\title{
The design of grinding wheel balancing control system and control software
}

\author{
Chao Liu ${ }^{a}$, Wufa Liu ${ }^{b}$ and Chunlei Mei ${ }^{\mathrm{c}}$ \\ School of Mechanical Engineering, Zhengzhou University, Zhengzhou 450001, China \\ achaoc888@163.com, bwufaliu126@126.com, '595263426@qq.com
}

Keywords: grinding wheel, dynamic balancing, and signal processing, automatic control.

\begin{abstract}
In order to reduce the unbalance of grinding wheel influence on machining accuracy and the life of grinding machine, a new grinding wheel dynamic balancing control system has been designed, it contains hardware and software part of the system. The system is based on ARM (STM32F417) as the core processor, it consists of the vibration measurement module, rotational speed measurement module, signal processing module, balancing control module and so on. The system can real-time monitor the unbalance through piezoelectric sensor when grinding wheel is rotating, then the drive control circuit of control card controls two eccentric gears rotate in balance head to achieve grinding wheel balancing. The system has high efficiency and can improve machining accuracy of grinder.
\end{abstract}

\section{Introduction}

Because of the uneven distribution of wheel structure, Geometric imperfections, assembly error and other reasons may cause the centroid of grinding wheel deviate from center of rotation [1]. It can produce the unbalanced centrifugal force and cause vibration of grinding wheel. The vibration will produce noise and accelerate abrasion of bearings, which can seriously affect the grinding accuracy and the service life of grinding machine [2]. The accuracy of traditional manual static balancing is very low. It has not taken the assembly error and vibration of other components into consideration. So it can not satisfy the requirement of high precision machining. The online automatic dynamic balance technology can compensate for grinding wheel unbalance efficiently and accurately. With the rapid development of grinding technology, the online dynamic balance can improve the machining accuracy of grinding machine significantly [3]. So it is very important to design an automatic balancing control system with high efficiency, high precision and cheap which has great value in application.

\section{Overall system design}

Grinding wheel dynamic balancing control system mainly consists of vibration sensor, speed sensor, cable, balancing head, dynamic balancing control card and STM32F417 core processor. We use piezoelectric acceleration sensor to measure the magnitude of vibration and hall speed sensor to measure the rotating speed of the grinding wheel [4]. The balancing head is fixed on the end of grinding wheel spindle through flange and coupling nut. Figure 1 is a schematic diagram of the balancing head installation.

The functional block diagram of wheel dynamic balancing control system is as shown in Figure 2 . It use ARM processor as the core processing chip of the system. The measurement of amount of unbalance is through piezoelectric acceleration sensor when grinding wheel is rotating. It can make the physical quantity of vibration into voltage signal. We use hall sensor to measure the speed of grinding wheel, the speed signal can be used as a reference signal to measure phase. These signals are processed by the signal processing unit of the control card in order to obtain the true vibration and speed signals. The STM32F417 core module can do A/D conversion and data processing to get the amplitude and phase of amount of grinding wheel unbalance. When the amplitude of amount of unbalance exceeds a predetermined value, the drive control circuit controls the rotation of two DC 
motors to change two partial tooth circles' position. Finally the grinding wheel realized dynamic balance.

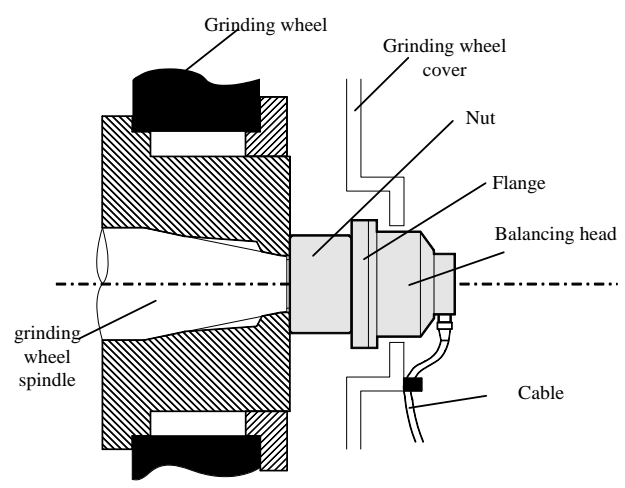

Fig. 1 Assembly drawing of balancing head

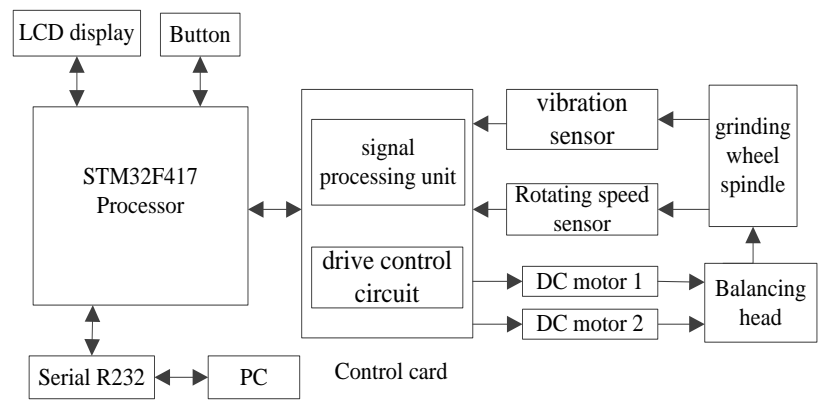

Fig. 2 Block diagram of wheel balancing system

\section{Hardware design of control system}

\subsection{Design of signal processing circuit.}

The signal from the acceleration sensor is weak and contains some noise signal. The analog signal is aperiodic random signals which contains vibration signal of grinding wheel and other kinds of vibration signal. So it must be processed through signal processing circuit of control card to get the true vibration signal. Vibration signal processing circuit includes preamplifier circuit, integrating circuit, programmable amplifier, band-pass filter circuit, phase-sensitive detection circuit, etc.

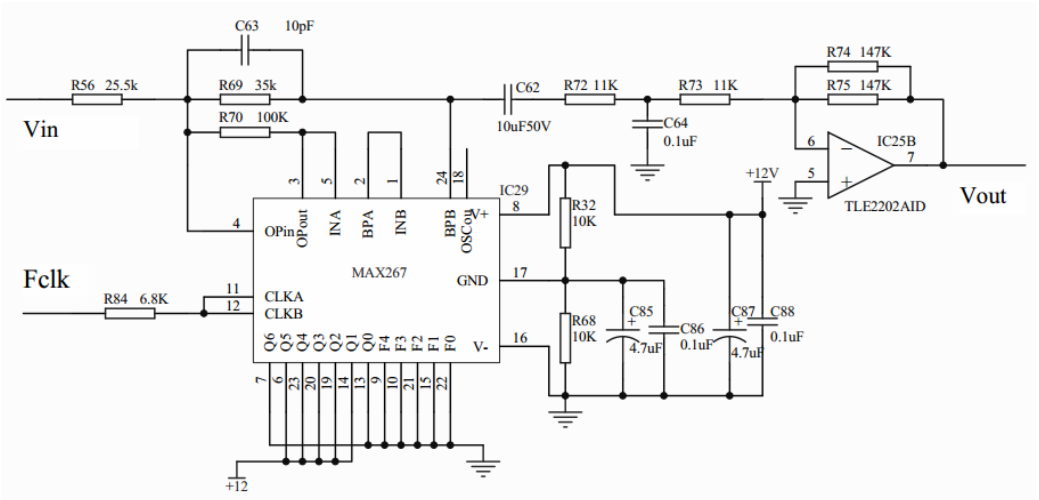

Fig. 3 Band-pass filtering and amplifying circuit of vibration signal

Figure 3 is the band-pass filtering and amplifying circuit of vibration signal. The actual vibration signal is a mixed signal which contains a variety of frequency components. Because the frequency of real vibration signal is the same as the frequency of rotating speed of grinding wheel, so the original signal must be processed by filter circuit to eliminate the noise signal. We choose the switched capacitor active filter MAX267 to realize the function of band pass filtering. The ratio between clock frequency and the center frequency is selected via programmable pins F0-F4. The Q is set up via pins 
Q0-Q7. Their specific connection diagram is as shown in figure 4. Q=0.969. Fclk: f0=100:1. We input a clock in pin 18 which's frequency is 100 times than the frequency of rotation speed. Then we can keep the center frequency of the filter is consistent with the speed of the same frequency.

\subsection{The design of drive control circuit.}

Drive control circuit is designed to control the movement of two DC motors in balancing head. Then they control the movement of two eccentric gears through worm gear drive system to achieve grinding wheel balance compensation.

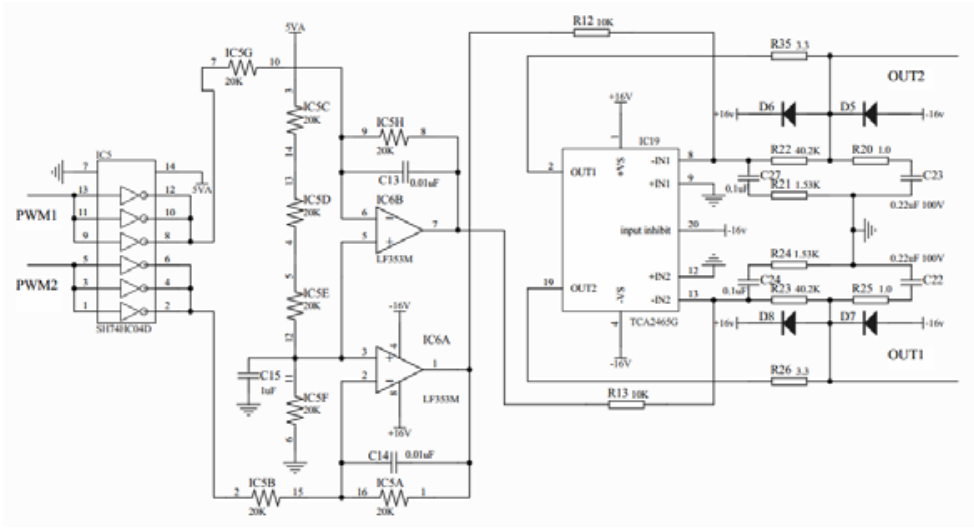

Fig. 4 Drive control circuit

Figure 4 is design of the drive control circuit. This paper adopts PWM to control the operation of DC motor. Two PWM signals are generated by general timer TIM2 and TIM3. We can set the TIMx-ARR register by software to determine the frequency of PWM signal. Pulse duty ratio of PWM can be changed by TIMx-CCRx. The PWM signals from STM32F417 are processed through inverter SH74HC04D. Its purpose is to enhance the driving capability of signals. The main control chip is TCA2465G in driver control circuit. It is dual power operational amplifier. The output peak current for TCA2465G is $2 \mathrm{~A}$ and large supply voltage range up to 42V. It do power amplify of control signal to control the DC motors. We adjust PWM duty ratio to change the output voltage magnitude of voltage [5]. It can be positive voltage or negative voltage. So DC motors can rotate forward or reverse. Then two partial tooth circles' positions are changed.

\section{The design of control software}

Design of the software has clear structure which can be divided into several modules, this system software mainly contains system initialization, vibration measurement module, speed measurement module, signal processing module, balance control module, display module, etc. The flow chart of software for grinding wheel balancing control system is shown in figure 5 .

There are two ways for the balance of the system, automatic balance and manual balance which can be selected by the buttons. Coordinate rotation method is used in the process of automatic balance when it comes to the control strategy [6]. The balancing process can be divided into two steps which include adjustment of phase coming first and adjustment of amplitude next. The detail Interpretation of balancing process is shown as follows: at first, in order to adjust the phase, two eccentric gears must have a fixed angle and then circle by same direction around the axis of the grinding wheel. But if the vibration amplitude shown on the screen is bigger than before, two eccentric gears must circle in opposite direction until the vibration amplitude doesn't become bigger any more. So the second steps can be implemented then. This time, two eccentric gears need circle by a direction of back-to-back, while f the vibration amplitude shown on the screen is bigger than before, two eccentric gears must circle in opposite direction until the vibration amplitude doesn't become bigger any more. As for manual balance, the difference is ways of controlling the two eccentric gears which can circle according to the signal from the related buttons [7]. The only purpose is guaranteeing the balance precision which can meet the requirement of the system. 


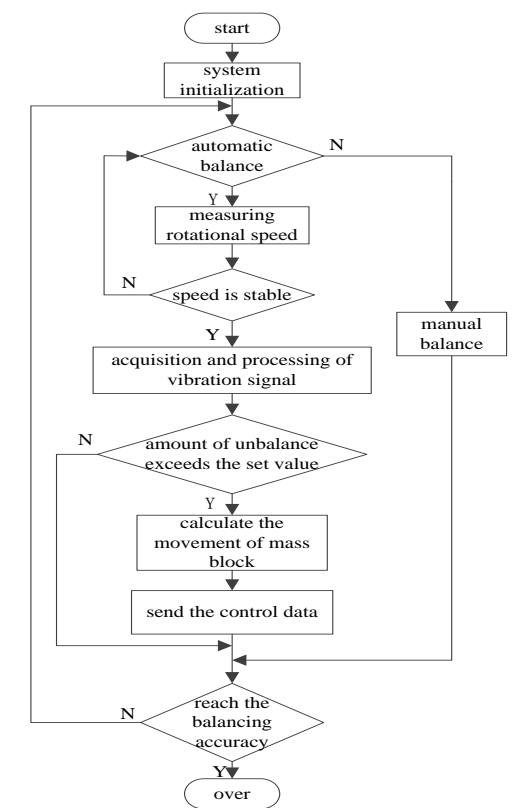

Fig. 5 Software flowchart of balancing control

\section{Conclusion}

This paper introduces the design process of hardware and software of a new grinding wheel dynamic balancing control system. System can effectively remove the interference signal through hardware circuit and get the real vibration signal. Then we measured the size of the amplitude and phase of vibration. The drive control circuit can control the rotation of DC motor efficiently and stably. This system is simple to operate; the equilibration time is short, high balancing accuracy and efficiency. It can significantly improve the machining accuracy.

\section{References}

[1]. Libo Tian, Dapeng Dong. Measurement and control System for grinding wheel dynamic balance base on DSP. Science and Technology Innovation Herald. (2010) No. 33, p. 121.

[2]. Xia Zhang, Xiantao Yu, Qinglei Du. Realization of Dynamic Balancing Control of the Emery Wheel. Vol. 37(2008) No. 4, p. 32-34.

[3]. Js Rao. Vibration Condition Monitoring of Machine. Pangbourne Alph Scientist International Ltd. Vol. 1(2000) No. 3, p. 12-13.

[4]. Yie Zhong. Rotor dynamics. Tsinghua University Press, 1987, p. 237-252.

[5]. Jianhua Tan, Development of the rotor dynamic balancing testing system (, Jiangsu University, China 2010). P. 38-42

[6]. Xiaohong Yang, Wenchao Jia, Wen You. Wavelet analysis algorithm applied in grinding wheel dynamic signal. Computer Automated Measurement \& Control. Vol. 12 (2004) No. 10, p. 979-983.

[7]. Federn, K. Multi-plane Balancing of Elastic Rotors. Fundamental Theories and Practical Application. Vol. 12 (1997) No. 6, p. 143-207. 